\title{
In vitro study methodologies to investigate genetic aspects and effects of drugs used in attention-deficit hyperactivity disorder
}

\author{
Grünblatt, Edna ; Bartl, Jasmin ; Marinova, Zoya ; Walitza, Susanne
}

\begin{abstract}
Attention-deficit/hyperactivity disorder (ADHD) is one of the most common psychiatric disorders in children and adolescents, with up to $5 \%$ affected worldwide. Twin and family studies on ADHD show its high familiality with heritability estimated around $70 \%$, but, to date, no specific polymorphism or gene was found to be specifically affected. Psychostimulants (amphetamine, methylphenidate) and non-psychostimulants (atomoxetine) are used successfully in ADHD therapy, but many of their mechanisms of action and their adverse effects are not yet fully understood. Therefore, both genetic findings and therapeutic interventions should be further investigated. One easy platform for such studies is in vitro analyses, which encompass neuronal cell culture studies, transfections of genetic constructs, binding and electrophysiology analyses. In this review, different methods will be referred in particular to ADHD findings, and new techniques will be mentioned for future studies of drug or genetic effects in vitro.
\end{abstract}

DOI: https://doi.org/10.1007/s00702-012-0869-9

Posted at the Zurich Open Repository and Archive, University of Zurich

ZORA URL: https://doi.org/10.5167/uzh-74484

Journal Article

Accepted Version

Originally published at:

Grünblatt, Edna; Bartl, Jasmin; Marinova, Zoya; Walitza, Susanne (2013). In vitro study methodologies to investigate genetic aspects and effects of drugs used in attention-deficit hyperactivity disorder. Journal of Neural Transmission, 120(1):131-139.

DOI: https://doi.org/10.1007/s00702-012-0869-9 


\title{
$70^{\text {th }}$ Birthday Prof. Riederer
}

\section{In-vitro study methodologies to investigate genetic aspects and effects of} drugs used in Attention-deficit Hyperactivity Disorder

\author{
Grünblatt Edna $^{凶, 1}$, Bartl Jasmin ${ }^{2}$, Marinova Zoya ${ }^{1}$, Walitza Susanne ${ }^{1}$ \\ ${ }^{1}$ Department of Child and Adolescent Psychiatry, University of Zurich, Neumuensterallee 9, \\ 8032 Zurich, Switzerland \\ ${ }^{2}$ Clinical Neurochemistry, National Parkinson Foundation Centre of Excellence Laboratories, \\ Clinic for Psychiatry, Psychosomatic and Psychotherapy, University of Würzburg, Germany
}

Page No.: 26 Figures: 0 Tables: 0 Abstract word count: 142 words Total word count: 4738 words

${ }^{\bowtie}$ Corresponding author: Grünblatt Edna, Ph.D.

Department of Child and Adolescent Psychiatry

University of Zurich

Thurgauerstrasse 39

CH-8050 Zürich / Switzerland

Tel: +41(0)44578 6071

Fax: +41(0)44 5786081

Email: edna.gruenblatt@kjpdzh.ch 


\begin{abstract}
Attention-deficit/hyperactivity disorder (ADHD) is one of the most common psychiatric disorders in children and adolescents, with up to $5 \%$ affected worldwide. Twin and family studies on ADHD show its high familiality with heritability estimated around $70 \%$, but, to date, no specific polymorphism or gene was found to be specifically affected. Psychostimulants (amphetamine, methylphenidate) and non-psychostimulants (atomoxetine) are used successfully in ADHD therapy, but many of their mechanisms of action and their adverse effects are not yet fully understood. Therefore, both genetic findings and therapeutic interventions should be further investigated. One easy platform for such studies are in vitro analyses, which encompass neuronal cell culture studies, transfections of genetic constructs, binding and electrophysiology analyses. In this review, different methods will be referred in particular to ADHD findings, and new techniques will be mentioned for future studies of drug or genetic effects in vitro.
\end{abstract}

Key Words: Atomoxetine, attention-deficit hyperactivity disorder, cell culture, electrophysiology, methylphenidate, neuron 


\section{Introduction}

Attention-deficit/hyperactivity disorder (ADHD) is one of the most common psychiatric disorders in children and adolescents, with up to $5 \%$ affected worldwide and with similar prevalence rates throughout different cultural settings (Polanczyk et al. 2007). ADHD is characterised as a clinically heterogeneous neurodevelopmental syndrome by the constitutive development of inappropriate levels of inattention, hyperactivity, and impulsiveness. The syndromal dimensions of inattention and enhanced impulsivity are increasingly recognised as highly persistent into adulthood and are associated with considerable risk for psychiatric comorbidity such as depression, substance abuse disorder, and failure in psychosocial adaptation (Jacob et al. 2007).

State-of-the-art treatments for ADHD include parental training, psychotherapeuticbehavioural therapy, and social interventions. According to the European Network for Hyperkinetic Disorders (EUNETHYDIS) Guideline Group, in severe cases and impairment, the use of medication is recommended (Banaschewski et al. 2008). Medications currently licensed for the treatment of children and adolescents with ADHD include psychostimulants such as methylphenidate hydrochloride (MPH) and dexamfetamine sulphate, and as second choice the non-stimulant atomoxetine (ATX). MPH, which reduces the core symptoms of the disorder and improves patients' quality of life, is the first-line treatment for ADHD. Stimulant medications are usually titrated until reaching optimal symptom reduction or until significant stimulant side effects occur. In the European guidelines of medication for ADHD (Graham et al. 2011) it is summarized that side effects occur, but they are generally acceptable because they are mild and/or temporary. MPH has a neuropharmacological profile similar to amphetamine and cocaine, which are well known as indirect dopamine (DA) agonists that cause a striatal DA overflow, probably via DA transporter (DAT) inhibition and partial norepinephrine transporter (NET) inhibition (Challman and Lipsky 2000). ATX, a non- 
psychostimulant, is known to act via norepinephrine (NE) reuptake inhibition (Dell'Agnello et al. 2009). However, a need for further clarification of the mechanisms of action of the various drug therapies still exists.

The cause and pathophysiology of ADHD is still unknown, but there is clear evidence that ADHD has multiple aetiologies (e.g. an interaction of genetic, environmental, neurobiological, and neurochemical factors) (Purper-Ouakil et al. 2011). ADHD shows high heritability as documented in family, twin, and adoption studies, with estimates of up to a 70\% heredity rate (Faraone and Doyle 2001). To date, although several genome-wide association studies (GWAS) and meta-analyses for association studies were conducted, no one single nucleotide polymorphism (SNP) or gene was found to be specifically affected in ADHD (Zhang et al. 2012). Still, it seems that dopaminergic, serotonergic, noradrenergic, synaptic, and growth factors genes are involved in ADHD. Therefore, some functional mechanism of action of such genetic findings might reveal the etiopathology of ADHD.

In vitro studies are one possible route in the quest to find the etiopathology of ADHD as well as the mechanism of action of its drug therapy. In vitro analyses span can be applied to cell culture studies of vesicular and synaptic release, binding, and electrophysiology analysis. In this review, different methods will be applied to ADHD findings, and new techniques will be mentioned for future studies of drug or genetic effects in vitro.

\section{Cell culture studies}

Several studies have been conducted on cell cultures, either non-neuronal transfected cultures or neuronal cells (primary/ cell lines) and astrocytic cells in order to elucidate the effects of drug therapy or the function of the transporter/ receptor thought to be involved in ADHD. Most of these studies involve toxicity /cell viability tests as well as morphological analysis of the cells. 


\section{Non-neuronal culture}

The toxicity effect of MPH was tested on non-neuronal human embryonic kidney (HEK-293) cells and HEK-293 cells stably transfected with the human DAT gene (HEK-hDAT) as a model limited to the inhibiting effects of MPH on DAT (Ludolph et al. 2006). Ludolph and colleagues demonstrated that no toxicity effect could be observed in both HEK-293 and HEKhDAT cells treated with MPH at doses of $1-1000 \mu \mathrm{M}$, as measured with an MTT assay (2006). In addition, they showed that MPH $(250-1000 \mu \mathrm{M})$ reduced cytotoxicity caused by 1-methyl4-phenylpyridinium $\left(\mathrm{MPP}^{+}\right)$in HEK-hDAT cells. In a later study by Schmidt et al., the effect of psychostimulants and ATX on cell survival of immune (monocytic U-937) cells was tested (2010a). Similar to Ludolph et al.'s study, Schmidt et al. also found increased cell survival, while ATP measurements for energy metabolism of the cells revealed no effect what-so-ever (2010a). Furthermore, Schmidt and colleagues tested whether psychostimulants and ATX affect the transcription of 8-hydroxyguanine glycosylase 1 (hOGG1), which catalyses the removal of 8-hydroxy-20-deoxyguanosine (8-oxo-OHdG), one of the predominant products of oxidative DNA lesions induced by reactive oxygen species (2010b). After U-937 cell treatment with various doses of amphetamine, MPH or ATX, they extracted total RNA for reverse-transcription polymerase chain reaction (RT-PCR) for the hOGG1 gene. They observed decreased expression of hOGG1 mRNA at a significant level after treatment with 5000ng/ml amphetamine as well as after treatment with 50, 500 and 5000ng/ml ATX (Schmidt et al. 2010b). Therefore, they concluded that these drugs play a protective role against oxidative DNA damage.

In order to learn the functional differences of DA or NE transport blockage, one could use transfected cells bearing the DAT or NET. The possibility of whether transporters can promote cellular adaptation, analogous to receptors, was raised. In HEK cell lines transfected with the hDAT, human DAT, its substrates (DA, amphetamine), and clinically relevant DAT 
inhibitors (cocaine, MPH and bupropion) reportedly increased c-Fos immunoreactivity (Yatin et al. 2002). To demonstrate this novel catecholamine transporter-dependent function, Yatin et al. developed an immunofluorescent method to quantify c-Fos in individual cells. Increased expression of the immediate early gene $c$-fos initiates a cascade of intracellular events that may underlie neuroadaptive changes following repeated exposure to the drugs. Yatin et al. investigated whether substrates (DA, NE) of the human DAT and NET can directly induce cFos protein in HEK cells transfected with hDAT and hNET and whether protein kinase $\mathrm{C}$ (PKC) modulators affect this process (2005). This group's demonstration that DA and NE can induce c-Fos expression in a monoamine transporter-dependent manner suggests the possibility that elevated catecholamine levels induced by psychostimulant drugs of abuse, such as cocaine and amphetamine, as well as clinically relevant medications such as MPH and bupropion, may affect gene expression and serve as a trigger for neuroadaptive responses in neurons expressing monoamine transporters (Yatin et al. 2005).

Transfected cell cultures are another useful tool in exploring the functional effects of known mutations associated with ADHD. For example, the 40-bp variable number of tandem repeats (VNTR) polymorphism in the $15^{\text {th }}$ exon of DAT1 (SLC6A3) (Vandenbergh et al., 1992a; Vandenbergh et al., 1992b) is known to reside in a region encoding the 3' untranslated region (3'-UTR), which does not alter the protein's amino acid sequence, but the 10-repeat variant is reportedly associated with ADHD (Nemoda et al. 2011). In a study in which HEK cells were transfected with four different DATl constructs (hDAT, DATl coding the region necessary and sufficient to produce a functional transporter protein; hDAT Zero, a construct containing the DAT1 coding region flanked by an $\sim 800 \mathrm{bp}$ fragment of the 3 '-UTR upstream of the VNTR region; hDAT 9, a construct with the DAT1 coding region upstream of a full length 3'UTR harbouring the 9-repeat VNTR; hDAT 10, a construct with the DAT1 coding region upstream of a full length 3'-UTR containing the 10-repeat VNTR), VanNess et al. examined 
the effects of the DAT1 VNTR on measures of in-vitro DAT expression and pharmacology (2005). They were able to demonstrate that DAT expression, as measured using binding assays and DAT immunoblotting, is attributed to the DAT1 genotype. Cells carrying the 10repeats DAT1 variants were characterised by a $\mathrm{B}_{\max }$ approximately $50 \%$ greater than cells with the 9-repeat VNTR; those containing only the DATl coding region or the coding region flanked by a truncated 3'-UTR resulted in greater DAT density than either of the naturalistic 9- and 10-repeat variants (VanNess et al. 2005). On the other hand, competition binding assays showed no statistically significant DAT1 genotype effects on the DAT affinity for MPH, as expected (VanNess et al. 2005).

In studying the transport of drugs into the central nervous system (CNS), Zhu et al. used the porcine kidney epithelial cell line LLC-PK1 and its human P-glycoprotein overexpressing mutant LLC-PK1/MDR1 cells (2008a). They investigated whether the P-glycoprotein, a member of the adenosine triphosphate-binding cassette transporter superfamily that has been found in a large number of normal tissues including intestine, liver, placenta, kidney, and the blood-brain barrier (BBB), interacts in the drug transport of ATX, MPH, amphetamine, and modafinil (Zhu et al. 2008a). Since it is known that in the BBB, P-glycoprotein prohibits substrate drug penetration into the CNS (Mayer et al. 1997; van Asperen et al. 1997), Zhu and colleagues used intracellular uptake studies with fluorescent substrates doxorubicin and rhodamine123 as well as a cell monolayer culture of the transport efficiencies (Zhu et al. 2008a). Their results demonstrated that all tested agents with the exception of modafinil isomers are relatively weak P-glycoprotein inhibitors. Furthermore, P-glycoprotein may play a minor role in the transport of d-MPH, d-modafinil, and 1-modafinil. The same research group established another cell line that stably expressed specific mutation of the human carboxylesterase (CES)1 (Zhu et al. 2008b). CES1 is the principal enzyme governing the metabolism of MPH and is involved in the metabolism of numerous other therapeutic 
medications as well as some illicit drugs such as heroin and cocaine. The mutation in exon 4 of CES1 is located in codon 143 and leads to a non-conservative substitution (Gly143Glu), which results in a reduction of enzyme activity and complete loss of hydrolytic activity toward methylphenidate. This specific CES1 mutation was also investigated in a Hungarian ADHD group (Nemoda et al. 2009), but no genotype effect could be shown. However, the group of Nemoda could detect that carrying the Glu-allele required lower doses of MPH for symptom reduction and so it seems that the mutation has an impact on MPH response and should be further investigated in larger ADHD samples.

\section{Neuronal culture}

Similar to the non-neuronal cell culture, the toxic effect of MPH was also tested on primary cultures from rat rostral mesencephalic tegmentum (Ludolph et al. 2006). An MTT assay demonstrated no toxicity effects of MPH on the neurons, and an additional immunocytochemistry and morphological analysis proved no effects on the dopaminergic neurons (measured as tyrosine-hydroxylase (TH) positive neurons) (Ludolph et al. 2006). MPH has also proved protective effects against $\mathrm{MPP}^{+}$in this cell culture, a result similar to the non-neuronal culture (Ludolph et al. 2006). Again, Schmidt et al. tested various psychostimulants and ATX for their toxicity effect on human neuroblastoma SH-SY5Y cells (2010a). As with the non-neuronal cell culture, MPH and ATX also enhanced cell survival (Schmidt et al., 2010a). As described above, in the non-neuronal cell culture, Schmidt et al. also demonstrated a decreased hOGG1 mRNA expression after treatment with psychostimulants or ATX of the SH-SY5Y cells (2010b).

Recently, our study group tested the effects of MPH on dopaminergic neuronal cell culture devoid of DAT (Bartl et al. 2011). Through this system, we could test MPH effects on the cells that are not associated with DAT inhibition. For this study, we used rat pheochromocytoma cells (PC12) that do not express DAT, but do express NET. MPH's 
influence in a dose response was tested for neurotransmitter levels (release, metabolism, and cytoplasmic) using high performance liquid chromatography (HPLC). Gene expression levels of synaptic genes were tested using the quantitative RT-PCR (Q-PCR), and cell proliferation studies using bromodeoxyuridine (BrdU) were conducted. Treatment with low-dose MPH (1$100 \mathrm{nM}$ ) altered intra-/extracellular neurotransmitter levels, down-regulated all investigated genes (NET (SLC6A2), synaptotagmin 1 and 4, syntaxin 1a, and synaptic vesicle glycoprotein 2C), and significantly enhanced cell proliferation. These data point to the diverse effects of MPH on cell metabolism independent of inhibiting DAT (Bartl et al. 2011). This study did not investigate the effects of MPH on other uptake systems, for example, organic cation transporters (OCT) or plasma membrane monoamine transporter (PMAT). Dwas et al. reported in detail that in addition to traditional uptake systems like DAT, NET, or serotonin transporter (SERT), OCT and/or PMAT are capable of clearing biogenic amines from extracellular fluid and may serve to buffer the effects of frontline antidepressants, MPH and amphetamine (Dwas et al. 2009). The role of OCT in the mechanism of action of MPH is not known so far, but it seems to be a very important aspect and, in addition to DAT inhibition, should be taken into account for future MPH studies.

The internalization and recycling of the DAT on the membrane surface was studied since PKC was demonstrated to down-regulate DA transport, primarily by redistributing the DAT from the plasma membrane to endosomal compartments, which might play an important role in treatment with psychostimulants (Loder and Melikian 2003). For this purpose, Loder and Melikian used PC12 cells that stably expressed the human DAT (DAT-PC12). For the effects of DAT internalisation and recycling, they used cell surface biotinylation and then SDSpolyacrylamide gel electrophoresis and immunoblotting. In this study, they demonstrate constitutive DAT trafficking, and that PKC-mediated DAT sequestration is achieved by a combination of accelerated internalisation and reduced recycling (Loder and Melikian 2003). 


\section{Astrocytes and glial culture}

Since it was reported that astrocytes, which are the most numerous glial cells in the CNS, play an important role in the development of synaptic plasticity induced by methamphetamine (Miyatake et al. 2005; Narita et al. 2005; Narita et al. 2006), Suzuki et al. investigated whether treatment with MPH could affect the morphology of astrocytes, compared to methamphetamine (Suzuki et al. 2007). Here, they used limbic neuron/glia co-cultures from newborn mice that were treated in a dose response study with MPH or methamphetamine for 3 days. The effects on activated astrocytes were visualised using fluorescence immunocytochemistry with a mouse polyclonal antibody against glial fibrillary acidic protein (GFAP). From this study, it can be concluded that treatment with methamphetamine, but not MPH, caused long-lasting astrocytic activation in limbic neuron/glia co-cultures. These findings suggest that, unlike methamphetamine, MPH induces reversible astrocytic activation after washout (Suzuki et al. 2007). Narita and colleagues investigated the involvement of phosphatidylinisitol-3 kinase (PI3-K) in astrocyte activation (Narita et al 2009). Administration of a selective PI3-K inhibitor wortmannin, together with methamphetamine or MPH, markedly and significantly inhibited methamphetamine-/MPH-induced astrocyte activation, suggesting the involvement of PI3-K in this process. In primary neuron/glia cocultures, a high concentration of methamphetamine dose-dependently reduced the number of cells positive for microtubule associated protein $2 \mathrm{a} / \mathrm{b}$ (MAP2a/b), which is a marker for neuronal dendrites; in contrast, MPH treatment did not change the number of MAP2a/bpositive cells. Narita et al. then measured the immunoreactivity of cleaved caspase-3, which is an activated form of apoptosis-related protein caspase-3, in order to reveal whether the reduction of MAP2a/b positive cells is due to apoptosis (Narita et al. 2009). They found that a high concentration of methamphetamine induced cleaved caspase-3, whereas the same treatment with MPH did not induce cleaved caspase-3. The above-mentioned homology 
between the reduction of MAP2a/b-positive cells and the induction of cleaved caspase-3 implies that a high concentration of methamphetamine in primary neuron/glia co-cultures causes neuronal cell death. In contrast, MPH lacks such an effect.

Recently, since NE activation of $\beta$-adrenergic receptors has been observed to induce the synthesis of the chemokine monocyte chemoattractant protein (CCL2/MCP-1) in astrocytes, the mechanisms involved in this process, particularly the possible effect of NE modulating drugs, was tested (Hinojosa-Arango et al. 2009). In this study, the authors used primary rat astrocyte cultures treated with NET inhibitors, either desipramine or ATX. The expression and synthesis of CCL2/MCP-1 in these cells were investigated using Q-PCR, enzyme-linked immunosorbent assay (ELISA), and immunocytochemsitry methodologies. Both drugs induced an increase in CCL2/MCP-1 expression in astrocytes (Hinojosa-Arango et al. 2009). These results suggest that CCL2/MCP-1 expression could also be modulated by some mechanism independent of the elevation of brain NE levels. This was confirmed by measuring a reduction in CCL2/MCP-1 production by treatment with clonidine, a $\alpha 2$ adrenergic receptor agonist.

\section{Drug binding and transport inhibition}

Since many of the ADHD drug therapies involve inhibition of synaptic DA or NE uptake, binding and uptake inhibition potency assays are an important part of drug-effect studies. One possibility for such an investigation is using either cell cultures that harbour the transporter of interest or using stably transfected cells. These are then used for an uptake assay with a radioactive marked substance or for a ligand binding assays using a radioligand and a nonradioactive competitor. Such study was conducted by Ukairo et al., who investigated the influences on DAT function at the level of the cultured cell by addressing the following: the age of the cell line (measured by cell passage number), the density of the cell monolayer (i.e., percent confluence), and the effect of varying DAT expression levels by manipulation of 
transfection conditions (Ukairo et al 2007). To ensure that comparisons between DA uptake inhibition potency (DUIP) and apparent binding affinity were legitimate for a given DAT inhibitor, $\left[{ }^{3} \mathrm{H}\right]-\mathrm{DA}$ uptake assays, binding assays involving the cocaine analogue $\left[{ }^{3} \mathrm{H}\right]-\mathrm{WIN}$ 35,428 , and versions of each assay that included competitor nonradioactive DAT blockers were conducted under identical conditions. To address, in part, the physiological relevance of such a phenomenon, neuronal and non-neuronal cell lines were similarly tested for DAT inhibitor DUIP fluctuations. Fluctuations in DUIP were observed for classical DAT blockers including cocaine, mazindol, MPH, and benztropine in $\mathrm{CHO}$ cells expressing wild type DAT (Ukairo et al. 2007). Cocaine potency also decreased in DAT-expressing non-neuronal COS-7 cells and murine neuroblastoma (N2A) cells (Ukairo et al. 2007). In contrast, the DAT substrate amphetamine did not display this DUIP fluctuation. In parallel experiments, no fluctuation was observed for the apparent binding affinities of these 5 drugs. The DUIP decrease appeared to correlate with an increase in cell surface DAT expression level, as measured by $\mathrm{B}_{\max }$ values and confocal microscopy. The fact that the DUIP profile of amphetamine diverged from that of the classical DAT blockers is consistent with the idea of fundamental differences between the mechanisms of abused psychostimulant DAT substrates and inhibitors (Ukairo et al. 2007).

Such binding and affinity study was also able to demonstrate the differences between the two isomers of MPH, the $d$ - and $l$-threo-isomers (Markowitz et al. 2006; Markowitz and Patrick 2008). Markowitz et al. demonstrated that MPH has affinity for the serotonergic 5-HT1A and 5-HT2B receptor sites for both $d$ - and $l$-MPH, with $d$-MPH exerting by far the most predominant effects $(2006 ; 2009)$. In addition, they showed a marked difference in affinity to NET between the $d$ - and $l$-isomers, of which $d$-MPH exhibited prominent affinity. 


\section{Electrophysiology}

Electrophysiological studies, either on brain slices or single cells using patch clamp with electro- or chemical- excitation, are useful tools to investigate the inhibition/activation of drugs such as MPH and ATX. For example, MPH was shown to increase cortical excitability via activation of $\alpha 2$-noradrenergic receptors (Andrews and Lavin 2006). Similarly, in another study, it was demonstrated that the MPH-induced response is probably mediated by NE via $\alpha 2 \mathrm{~B} / 2 \mathrm{C}$-adrenoceptors in locus coeruleus neurons (Ishimatsu et al. 2002). In a later study, whole-cell patch-clamp recordings revealed that MPH enhances inhibitory synaptic transmission by increasing the concentration of $\mathrm{NE}$ at noradrenergic synapses in juvenile rat locus coeruleus neurons (Kidani et al. 2010). On the other hand, in the dopaminergic neurons originating from the ventral tegmental areas, Prieto-Gomez and colleagues hypothesised after electrophysiological study on brain slices that psychostimulants sensitisation could be involved, though both N-Methyl-D-aspartate (NMDA) and kainite/AMPA glutamate receptors medicated the excitability of these neurons (Prieto-Gomez et al 2004; 2005).

ATX has also been studied for its mechanism of action using electrophysiological methods. Kobayashi and colleagues investigated the effects of ATX and reboxetine on G-proteinactivated inwardly rectifying $\mathrm{K}^{+}$(GIRK) channels using the Xenopus oocyte expression assay (Kobayashi et al 2010). In oocytes injected with mRNA for GIRK1/GIRK2, GIRK2, or GIRK1/GIRK4 subunits, an extracellular application of ATX or reboxetine reversibly reduced GIRK currents. The inhibitory effects were concentration dependent, but voltage-independent, and time-independent during each voltage pulse (Kobayashi et al. 2010). Such findings were suggested to point to the therapeutic effects of NET inhibitors and their adverse side effects related to the nervous system and heart function. In addition, a recent publication by Ludolph et al. demonstrated effective NMDA receptor antagonism by ATX at low micromolar concentrations in different cell culture models (Ludolph et al 2010). Therefore, it was 
proposed that the antagonism at the NMDA receptors by ATX might contribute to the clinical effect in ADHD.

Studies of specific genetic mutations using electrophysiological techniques could also be useful in understanding the effects of such mutations on neurotransmitter efflux, as demonstrated by Bowton et al. (Bowton et al. 2010). They report that tonic activation of the $\mathrm{D}_{2}$ receptor provides support for hDAT A559V-mediated anomalous DA efflux. These studies identify a signalling network downstream of $\mathrm{D}_{2}$ receptor activation, normally constraining dopaminergic action at synapses that may be altered by DAT mutation to impact risk for ADHD.

\section{Future prospective and methods}

Recently, several new in vitro methods have emerged to be potentially useful in studies associated with ADHD genetic mechanisms of action or drug function. Such methods could provide additional knowledge for better understanding of the disorder and/or potential targets for new therapy developments.

\section{Stem cells}

Since ADHD is considered a neurodevelopmental disorder, understanding how genes function in human brain development and how they interact with each other to cause a psychiatric disorder would be of great value. Mouse and human stem cells can teach us a great deal about how typical differentiation programs are implemented and how they may be modified in disease. But since stem cells are usually derived from embryos, the work with human stem cells has been hampered by ethical concerns. One alternative to embryonic stem cells is tissue-specific stem cells from adult humans. Stem cells of the central nervous system, from the subventricular zone of the cerebral cortex and the subgranular zone of the dentate gyrus, are of obvious interest for neuropsychiatric disorders. But again, this is quite limited because to harvest such cells, one needs fresh tissue, usually obtained from biopsies. Recently, the 
enormous advantages of induced pluripotent stem cells (iPSCs), commonly derived from skin fibroblasts, have been discussed (see Review, Vaccarino et al. 2011). The fact that such cells are derived from a living person using a non-controversial cell type, are generated from a specific individual, maintain his/her genetic constitution and diversity, and can represent a source of differentiated cell types genetically compatible with the person of origin has great advantage. Specifically, examination of iPSCs from typically developing individuals will reveal how basic cellular processes and genetic differences contribute to individually unique nervous systems. Moreover, by comparing iPSCs from typically developing individuals and patients, differences at stem cell stages, through neural differentiation, and into the development of functional neurons may be identified that will reveal opportunities for intervention. The application of such techniques to early onset neuropsychiatric disorders is still on the horizon but has become a reality of current research efforts as a consequence of the revelations of many years of basic developmental neurobiological science.

\section{Impedance-based method of cell proliferation /differentiation monitoring (xCELLigence)}

To date, the cell culture studies for cell viability, cell death, proliferation, or differentiation rely on endpoint analysis. Thus, one of the major limitations in the interpretation of such analyses in time course is derived from the fact that extensive and laborious endpoint measurements in separate cultures are required to cover a sufficient amount of time points for interpretation of the kinetic. Therefore, such limitations of standard endpoint assays in vitro may be overcome using an impedance-based analysis system for the detection of cell viability (xCELLigence, Roche). Consequently, cell populations that differ in cell density, proliferation rate, adhesion characteristics, or cell morphology can be distinguished by the impedance readout (Xiao et al. 2002). Recently, this system was also tested for detection of neuronal cell death and compared to measurements of endpoint viability (Diemert et al. 2012). They could validate the xCELLigence system for real-time detection of cell death in a neuronal cell line 
of immortalised hippocampal neurons (HT-22 cells), neuronal progenitor cells (NPC), and differentiated primary cortical neurons (Diemert et al. 2012). They found a good correlation between impedance measurements and endpoint viability assays in HT-22 cells and NPC for detecting proliferation, cell death kinetics, and also the neuroprotective effects of pharmacological apoptosis inhibitors. However, in primary neurons, they could not detect dendritic outgrowth during differentiation of the cells. Cell death in primary neurons was detectable by the xCELLigence system; however, the changes in the cell index on the basis of impedance measurements depend to a great extent on the severity of the insult. Therefore, this new method presents great potential in studying cell cultures in a continuous real-time-course that can reveal the effects previously missed using conventional methods.

\section{Live-cell imaging}

The advantages of live-cell imaging have been discussed in many previous reviews (Meijering et al. 2009; Tsien 2003). The ability to visualise cells and subcellular dynamic processes in space and time has been made possible by revolutionary developments in imaging technology in the past two decades. Advances in molecular biology, organic chemistry, and materials science have resulted in an impressive toolbox of fluorescent proteins (GFP and variants) and nanocrystals (quantum dots) and have enabled the study of protein expression, localisation, conformation, diffusion, turnover, trafficking, and interaction (Giepmans et al. 2006; Lippincott-Schwartz and Patterson 2003). Hardware advances in optical systems design have taken light microscopy from wide field to (multiphoton) confocal and spinning disk microscopy (Stephens and Allan 2003), and more recent efforts to break the diffraction barrier have further extended the palette (Garini et al. 2005; Hell 2009). Together, these developments have redefined biological research by enabling the switch from fixed to living cells and from qualitative to quantitative imaging (Tsien 2003). 
The understanding of synaptic plasticity kinetics will advance neuroscience greatly in regard to understanding how neurons store information, how spatiotemporal patterns of synaptic input combined with postsynaptic neuronal activity produce neuronal plasticity, and how plasticity changes neuronal activity patterns (Kotaleski and Blackwell 2010). Live-cell imaging techniques together with new computer modelling may provide much higher resolution views into synaptic plasticity (Kotaleski and Blackwell 2010). Similarly, protein trafficking dynamics are now available using fluorescently tagged proteins, enabling visualisation of protein movements and correlation of a protein's dynamics with its changing structural state or ligand binding (Chamberlain and Hahn 2000). A newly developed technique is reported to observe DNA-methylation states in live-cell imaging using fluorescent probes that bind specifically to methylated DNA (Yamagata 2010). Such new aspects will allow the observation of epigenetic changes due to environmental alterations with time, which have not yet been available. Other techniques to visualise synaptic vesicle recycling (Kakazu et al. 2012) or the exocytosis of membrane proteins (Hildick et al. 2012) were reported to be successful in live-cell imaging.

To date, few studies using live-cell imaging associated with ADHD have been conducted. Still, Bolan et al. investigated the role of two $\mathrm{D}_{2}$ receptor-linked signalling pathways (extracellular signal-regulated kinase (ERK1/2) and the PI3-K) in mediating the $\mathrm{D}_{2}$ receptor regulation of DAT using the fluorescent DAT substrate 4-(4-(dimethylamino)-styryl)-Nmethylpyridinium $\left(\mathrm{ASP}^{+}\right.$) in live-cell imaging techniques (Bolan et al. 2007). This study could show that an increase in DAT function is ERK1/2-dependent but PI3-K-independent, which therefore suggests the possibility of a direct physical interaction between DAT and the $\mathrm{D}_{2}$ receptor (Bolan et al. 2007). $\mathrm{ASP}^{+}$was also demonstrated to be feasible in analysing the function of the serotonin transporter in HEK and neuroblastoma cells (Oz et al. 2010). 


\section{Conclusion}

Since the aetiology of ADHD is not yet fully revealed, and only some genetic and environmental factors that affect the neurodevelopmental processes have been suggested, the importance of further research in this field is rather obvious. Psychostimulants (e.g., amphetamine, MPH) and non-psychostimulants (e.g., ATX) are used successfully in ADHD therapy, but many of their mechanism of action and their adverse effects are not yet fully understood. Therefore, both genetic findings and therapeutic effects should be further investigated. In conclusion, using in vitro systems to investigate the function of genes involved in ADHD or the mechanism of action of drug therapy is a very valuable tool in ADHD research. In addition, the new techniques mentioned in this article as well as new to come can enhance our understanding of many processes that have not yet been revealed.

\section{Acknowledgement}

This work was partially supported by the German Research Foundation (KFO-125) and the Swiss National Science Foundation (SNF). We wish to thank Naozumi Araragi for his help translating a reference from Japanese into English. 


\section{Reference}

Andrews GD, Lavin A (2006) Methylphenidate increases cortical excitability via activation of alpha-2 noradrenergic receptors. Neuropsychopharmacology 31:594-601.

Banaschewski T, Coghill D, Santosh P, Zuddas A, Asherson P, Buitelaar J, Danckaerts M, Dopfner M, Faraone SV, Rothenberger A, Sergeant J, Steinhausen HC, Sonuga-Barke EJ, Taylor E (2008) [Long-acting medications for the treatment of hyperkinetic disorders - a systematic review and European treatment guideline. Part 1: overview and recommendations]. Z Kinder Jugendpsychiatr Psychother 36:81-94; quiz 94-85.

Bartl J, Link P, Schlosser C, Gerlach M, Schmitt A, Walitza S, Riederer P, Grunblatt E (2011) Effects of methylphenidate: the cellular point of view. Atten Defic Hyperact Disord 2:225-232.

Bolan EA, Kivell B, Jaligam V, Oz M, Jayanthi LD, Han Y, Sen N, Urizar E, Gomes I, Devi LA, Ramamoorthy S, Javitch JA, Zapata A, Shippenberg TS (2007) D2 receptors regulate dopamine transporter function via an extracellular signal-regulated kinases 1 and 2-dependent and phosphoinositide 3 kinase-independent mechanism. Mol Pharmacol 71:1222-1232.

Bowton E, Saunders C, Erreger K, Sakrikar D, Matthies HJ, Sen N, Jessen T, Colbran RJ, Caron MG, Javitch JA, Blakely RD, Galli A (2010) Dysregulation of dopamine transporters via dopamine D2 autoreceptors triggers anomalous dopamine efflux associated with attention-deficit hyperactivity disorder. J Neurosci 30:6048-6057.

Challman TD, Lipsky JJ (2000) Methylphenidate: its pharmacology and uses. Mayo Clin Proc 75:711-721.

Chamberlain C, Hahn KM (2000) Watching proteins in the wild: fluorescence methods to study protein dynamics in living cells. Traffic 1:755-762. 
Daws LC (2009) Unfaithful neurotransmitter transporters: focus on serotonin uptake and implications for antidepressant efficacy. Pharmacol Ther 121:89-99.

Dell'Agnello G, Zuddas A, Masi G, Curatolo P, Besana D, Rossi A (2009) Use of atomoxetine in patients with attention-deficit hyperactivity disorder and co-morbid conditions. CNS Drugs 23:739-753.

Diemert S, Dolga AM, Tobaben S, Grohm J, Pfeifer S, Oexler E, Culmsee C (2012) Impedance measurement for real time detection of neuronal cell death. J Neurosci Methods 203:69-77.

Faraone SV, Doyle AE (2001) The nature and heritability of attention-deficit/hyperactivity disorder. Child Adolesc Psychiatr Clin N Am 10:299-316, viii-ix.

Garini Y, Vermolen BJ, Young IT (2005) From micro to nano: recent advances in highresolution microscopy. Curr Opin Biotechnol 16:3-12.

Giepmans BN, Adams SR, Ellisman MH, Tsien RY (2006) The fluorescent toolbox for assessing protein location and function. Science 312:217-224.

Graham J et al. (2011) European guidelines on managing adverse effects of medication for ADHD. Eur Child Adolesc Psychiatry 20:17-37.

Hell SW (2009) Microscopy and its focal switch. Nat Methods 6:24-32.

Hildick KL, Gonz 225 Lez-Gonz 225 Lez IM, Jaskolski FD, Henley JM (2012) Lateral Diffusion and Exocytosis of Membrane Proteins in Cultured Neurons Assessed using Fluorescence Recovery and Fluorescence-loss Photobleaching. J Vis Exp.

Hinojosa-Arango G, Maggs CA, Johnson MP (2009) Like a rolling stone: the mobility of maerl (Corallinaceae) and the neutrality of the associated assemblages. Ecology 90:517-528. 
Ishimatsu M, Kidani Y, Tsuda A, Akasu T (2002) Effects of methylphenidate on the membrane potential and current in neurons of the rat locus coeruleus. $\mathrm{J}$ Neurophysiol $87: 1206-1212$.

Jacob CP, Romanos J, Dempfle A, Heine M, Windemuth-Kieselbach C, Kruse A, Reif A, Walitza S, Romanos M, Strobel A, Brocke B, Schafer H, Schmidtke A, Boning J, Lesch KP (2007) Co-morbidity of adult attention-deficit/hyperactivity disorder with focus on personality traits and related disorders in a tertiary referral center. Eur Arch Psychiatry Clin Neurosci 257:309-317.

Kakazu Y, Koh JY, Ho KW, Gonzalez-Alegre P, Harata NC (2012) Synaptic vesicle recycling is enhanced by torsinA that harbors the DYT1 dystonia mutation. Synapse 66:453-464.

Kidani Y, Ishimatsu M, Akasu T (2010) Methylphenidate enhances inhibitory synaptic transmission by increasing the content of norepinephrine in the locus coeruleus of juvenile rats. Kurume Med J 57:29-38.

Kobayashi T, Washiyama K, Ikeda K (2010) Inhibition of G-protein-activated inwardly rectifying $\mathrm{K}+$ channels by the selective norepinephrine reuptake inhibitors atomoxetine and reboxetine. Neuropsychopharmacology 35:1560-1569.

Kotaleski JH, Blackwell KT (2010) Modelling the molecular mechanisms of synaptic plasticity using systems biology approaches. Nat Rev Neurosci 11:239-251.

Lippincott-Schwartz J, Patterson GH (2003) Development and use of fluorescent protein markers in living cells. Science 300:87-91.

Loder MK, Melikian HE (2003) The dopamine transporter constitutively internalizes and recycles in a protein kinase C-regulated manner in stably transfected PC12 cell lines. J Biol Chem 278:22168-22174. 
Ludolph AG, Schaz U, Storch A, Liebau S, Fegert JM, Boeckers TM (2006) Methylphenidate exerts no neurotoxic, but neuroprotective effects in vitro. J Neural Transm 113:19271934.

Ludolph AG, Udvardi PT, Schaz U, Henes C, Adolph O, Weigt HU, Fegert JM, Boeckers TM, Fohr KJ (2010) Atomoxetine acts as an NMDA receptor blocker in clinically relevant concentrations. Br J Pharmacol 160:283-291.

Markowitz JS, Patrick KS (2008) Differential pharmacokinetics and pharmacodynamics of methylphenidate enantiomers: does chirality matter? J Clin Psychopharmacol 28:S5461.

Markowitz JS, DeVane CL, Ramamoorthy S, Zhu HJ (2009) The psychostimulant d-threo$(\mathrm{R}, \mathrm{R})$-methylphenidate binds as an agonist to the 5HT(1A) receptor. Pharmazie 64:123-125.

Markowitz JS, DeVane CL, Pestreich LK, Patrick KS, Muniz R (2006) A comprehensive in vitro screening of d-, l-, and dl-threo-methylphenidate: an exploratory study. J Child Adolesc Psychopharmacol 16:687-698.

Mayer U, Wagenaar E, Dorobek B, Beijnen JH, Borst P, Schinkel AH (1997) Full blockade of intestinal P-glycoprotein and extensive inhibition of blood-brain barrier Pglycoprotein by oral treatment of mice with PSC833. J Clin Invest 100:2430-2436.

Meijering E, Dzyubachyk O, Smal I, van Cappellen WA (2009) Tracking in cell and developmental biology. Semin Cell Dev Biol 20:894-902.

Miyatake M, Narita M, Shibasaki M, Nakamura A, Suzuki T (2005) Glutamatergic neurotransmission and protein kinase $\mathrm{C}$ play a role in neuron-glia communication during the development of methamphetamine-induced psychological dependence. Eur J Neurosci 22:1476-1488. 
Narita M, Asato M, Shindo K, Kuzumaki N, Suzuki T (2009) [Differences in neuronal toxicity and its molecular mechanisms between methamphetamine and methylphenidate]. Nihon Shinkei Seishin Yakurigaku Zasshi 29:115-120.

Narita M, Miyatake M, Shibasaki M, Tsuda M, Koizumi S, Yajima Y, Inoue K, Suzuki T (2005) Long-lasting change in brain dynamics induced by methamphetamine: enhancement of protein kinase C-dependent astrocytic response and behavioral sensitization. J Neurochem 93:1383-1392.

Narita M, Miyatake M, Shibasaki M, Shindo K, Nakamura A, Kuzumaki N, Nagumo Y, Suzuki T (2006) Direct evidence of astrocytic modulation in the development of rewarding effects induced by drugs of abuse. Neuropsychopharmacology 31:24762488.

Nemoda Z, Szekely A, Sasvari-Szekely M (2011) Psychopathological aspects of dopaminergic gene polymorphisms in adolescence and young adulthood. Neurosci Biobehav Rev 35:1665-1686.

Nemoda Z, Angyal N, Tarnok Z, Gadoros J, Sasvari-Szekely M (2009) Carboxylesterase 1 gene polymorphism and methylphenidate response in ADHD. Neuropharmacology 57:731-733.

Oz M, Libby T, Kivell B, Jaligam V, Ramamoorthy S, Shippenberg TS (2010) Real-time, spatially resolved analysis of serotonin transporter activity and regulation using the fluorescent substrate, ASP+. J Neurochem 114:1019-1029.

Polanczyk G, de Lima MS, Horta BL, Biederman J, Rohde LA (2007) The worldwide prevalence of ADHD: a systematic review and metaregression analysis. Am J Psychiatry 164:942-948. 
Prieto-Gomez B, Benitez MT, Vazquez-Alvarez AM, Yang PB, Reyes Vazquez C, Dafny N (2004) Dopaminergic ventral tegmental neurons modulated by methylphenidate. Life Sci 74:1581-1592.

Prieto-Gomez B, Vazquez-Alvarez AM, Martinez-Pena JL, Reyes-Vazquez C, Yang PB, Dafny N (2005) Methylphenidate and amphetamine modulate differently the NMDA and AMPA glutamatergic transmission of dopaminergic neurons in the ventral tegmental area. Life Sci 77:635-649.

Purper-Ouakil D, Ramoz N, Lepagnol-Bestel AM, Gorwood P, Simonneau M (2011) Neurobiology of attention deficit/hyperactivity disorder. Pediatr Res 69:69R-76R.

Schmidt AJ, Krieg JC, Clement HW, Gebhardt S, Schulz E, Heiser P (2010a) Impact of drugs approved for treating ADHD on the cell survival and energy metabolism: an in-vitro study in human neuronal and immune cells. J Psychopharmacol 24:1829-1833.

Schmidt AJ, Clement HW, Gebhardt S, Hemmeter UM, Schulz E, Krieg JC, Kircher T, Heiser P (2010b) Impact of psychostimulants and atomoxetine on the expression of 8hydroxyguanine glycosylase 1 in human cells. J Neural Transm 117:793-797.

Stephens DJ, Allan VJ (2003) Light microscopy techniques for live cell imaging. Science 300:82-86.

Suzuki T, Shindo K, Miyatake M, Kurokawa K, Higashiyama K, Suzuki M, Narita M (2007) Lack of development of behavioral sensitization to methylphenidate in mice: correlation with reversible astrocytic activation. Eur J Pharmacol 574:39-48.

Tsien RY (2003) Imagining imaging's future. Nat Rev Mol Cell Biol Suppl:SS16-21.

Ukairo OT, Ramanujapuram S, Surratt CK (2007) Fluctuation of the dopamine uptake inhibition potency of cocaine, but not amphetamine, at mammalian cells expressing the dopamine transporter. Brain Res 1131:68-76. 
Vaccarino FM, Urban AE, Stevens HE, Szekely A, Abyzov A, Grigorenko EL, Gerstein M, Weissman S (2011) Annual Research Review: The promise of stem cell research for neuropsychiatric disorders. J Child Psychol Psychiatry 52:504-516.

van Asperen J, Mayer U, van Tellingen O, Beijnen JH (1997) The functional role of Pglycoprotein in the blood-brain barrier. J Pharm Sci 86:881-884.

Vandenbergh DJ, Persico AM, Uhl GR (1992a) A human dopamine transporter cDNA predicts reduced glycosylation, displays a novel repetitive element and provides racially-dimorphic TaqI RFLPs. Brain Res Mol Brain Res 15:161-166.

Vandenbergh DJ, Persico AM, Hawkins AL, Griffin CA, Li X, Jabs EW, Uhl GR (1992b) Human dopamine transporter gene (DAT1) maps to chromosome 5p15.3 and displays a VNTR. Genomics 14:1104-1106.

VanNess SH, Owens MJ, Kilts CD (2005) The variable number of tandem repeats element in DAT1 regulates in vitro dopamine transporter density. BMC Genet 6:55.

Xiao C, Lachance B, Sunahara G, Luong JH (2002) Assessment of cytotoxicity using electric cell-substrate impedance sensing: concentration and time response function approach. Anal Chem 74:5748-5753.

Yamagata K (2010) DNA methylation profiling using live-cell imaging. Methods 52:259-266.

Yatin SM, Miller GM, Madras BK (2005) Dopamine and norepinephrine transporterdependent c-Fos production in vitro: relevance to neuroadaptation. J Neurosci Methods 143:69-78.

Yatin SM, Miller GM, Norton C, Madras BK (2002) Dopamine transporter-dependent induction of C-Fos in HEK cells. Synapse 45:52-65.

Zhang L, Chang S, Li Z, Zhang K, Du Y, Ott J, Wang J (2012) ADHDgene: a genetic database for attention deficit hyperactivity disorder. Nucleic Acids Res 40:D10031009. 
Zhu HJ, Wang JS, Donovan JL, Jiang Y, Gibson BB, DeVane CL, Markowitz JS (2008a) Interactions of attention-deficit/hyperactivity disorder therapeutic agents with the efflux transporter P-glycoprotein. Eur J Pharmacol 578:148-158.

Zhu HJ, Patrick KS, Yuan HJ, Wang JS, Donovan JL, DeVane CL, Malcolm R, Johnson JA, Youngblood GL, Sweet DH, Langaee TY, Markowitz JS (2008b) Two CES1 gene mutations lead to dysfunctional carboxylesterase 1 activity in man: clinical significance and molecular basis. Am J Hum Genet 82:1241-1248. 